Network Working Group

Request for Comments: 5035

J. Schaad

Updates: 2634

Soaring Hawk Consulting

Category: Standards Track

August 2007

\title{
Enhanced Security Services (ESS) Update: \\ Adding CertID Algorithm Agility
}

Status of This Memo

This document specifies an Internet standards track protocol for the Internet community, and requests discussion and suggestions for improvements. Please refer to the current edition of the "Internet Official Protocol Standards" (STD 1) for the standardization state and status of this protocol. Distribution of this memo is unlimited.

Abstract

In the original Enhanced Security Services for S/MIME document (RFC 2634), a structure for cryptographically linking the certificate to be used in validation with the signature was introduced; this structure was hardwired to use SHA-1. This document allows for the structure to have algorithm agility and defines a new attribute for this purpose.

Table of Contents

1. Introduction . . . . . . . . . . . . . . . . . . . . . . 2

1.1. Notation . . . . . . . . . . . . . . . . . . . . . . 2

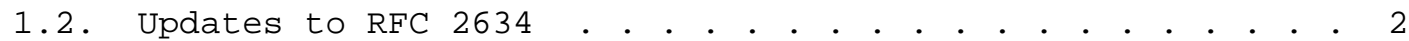

2. Replace Section 5.4'Signing Certificate Attribute Definitions' . . . . . . . . . . . . . . . . . . . . . 3

3. Insert New Section 5.4.1' Signing Certificate Attribute

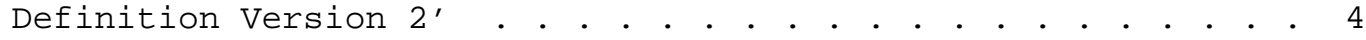

4. Insert New Section 5.4.1.1'Certificate Identification

Version 2' . . . . . . . . . . . . . . . . . . . . . . . . . 5

5. Insert New Section 5.4.2' 'Signing Certificate Attribute

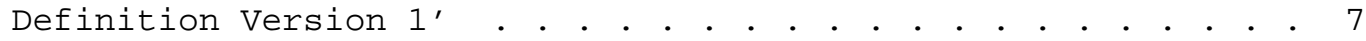

6. Insert New Section 5.4.2.1'Certificate Identification

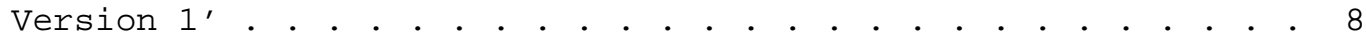

7. Security Considerations . . . . . . . . . . . . . . . . . . 9

8. Normative References . . . . . . . . . . . . . . . . 10

Appendix A. ASN.1 Module . • . . . . . . . . . . . . . . . . . 11 


\section{Introduction}

In the original Enhanced Security Services (ESS) for S/MIME document [ESS], a structure for cryptographically linking the certificate to be used in validation with the signature was defined. This structure, called ESSCertID, identifies a certificate by its hash. The structure is hardwired to use a SHA-1 hash value. The recent attacks on SHA-1 require that we define a new attribute that allows for the use of different algorithms. This document performs that task.

This document defines the structure ESSCertIDv2 along with a new attribute SigningCertificateV2, which uses the updated structure. This document allows for the structure to have algorithm agility by including an algorithm identifier and defines a new signed attribute to use the new structure.

This document specifies the continued use of ESSCertID to ensure compatibility when SHA-1 is used for certificate disambiguation.

\subsection{Notation}

The key words "MUST", "MUST NOT", "REQUIRED", "SHALL", "SHALL NOT", "SHOULD", "SHOULD NOT", "RECOMMENDED", "MAY", and "OPTIONAL" in this document are to be interpreted as described in [RFC2119].

\subsection{Updates to RFC 2634}

This document updates Section 5.4 of RFC 2634. Once the updates are applied, the revised section will have the following structure:

\subsection{Signing Certificate Attribute Definitions}

5.4.1 Signing Certificate Attribute Definition Version 2

5.4.1.1 Certificate Identification Version 2

5.4.2 Signing Certificate Attribute Definition Version 1

5.4.2.1 Certificate Identification Version 1

In addition, the ASN.1 module in Appendix A is replaced. 


\section{Replace Section 5.4 'Signing Certificate Attribute Definitions'}

\subsection{Signing Certificate Attribute Definitions}

The signing certificate attribute is designed to prevent simple substitution and re-issue attacks, and to allow for a restricted set of certificates to be used in verifying a signature.

Two different attributes exist for this due to a flaw in the original design. The only substantial difference between the two attributes is that SigningCertificatev2 allows for hash algorithm agility, while SigningCertificate forces the use of the SHA-1 hash algorithm. With the recent advances in the ability to create hash collisions for SHA-1, it is wise to move forward sooner rather than later.

When the SHA-1 hash function is used, the SigningCertificate attribute MUST be used. The SigningCertificateV2 attribute MUST be used if any algorithm other than SHA-1 is used and SHOULD NOT be used for SHA-1. Applications SHOULD recognize both attributes as long as they consider SHA-1 able to distinguish between two different certificates, (i.e., the possibility of a collision is sufficiently low). If both attributes exist in a single message, they are independently evaluated.

Four cases exist that need to be taken into account when using this attribute for correct processing:

1. Signature validates and the hashes match: This is the success case.

2. Signature validates and the hashes do not match: In this case, the certificate contained the correct public key, but the certificate containing the public key is not the one that the signer intended to be used. In this case the application should attempt a search for a different certificate with the same public key and for which the hashes match. If no such certificate can be found, this is a failure case.

3. Signature fails validation and the hashes match: In this case, it can be assumed that the signature has been modified in some fashion. This is a failure case.

4. Signature fails validation and the hashes do not match: In this case, it can be either that the signature has been modified, or that the wrong certificate has been used. Applications should attempt a search for a different certificate that matches the hash value in the attribute and use the new certificate to retry the signature validation. 
3. Insert New Section 5.4.1'Signing Certificate Attribute Definition Version 2'

\subsubsection{Signing Certificate Attribute Definition Version 2}

The signing certificate attribute is designed to prevent the simple substitution and re-issue attacks, and to allow for a restricted set of certificates to be used in verifying a signature.

SigningCertificateV2 is identified by the OID:

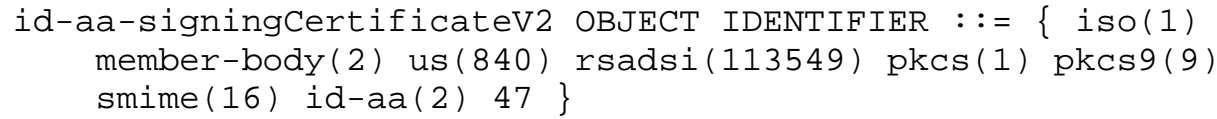

The attribute has the ASN.1 definition:

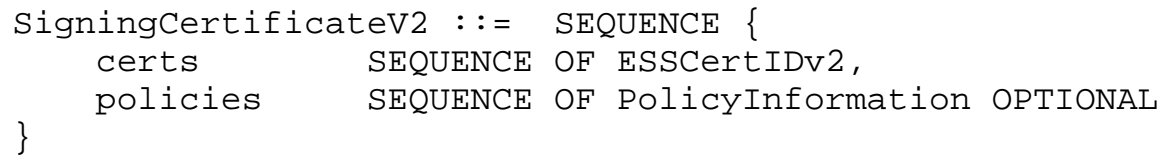


policies

contains a sequence of policy information terms that identify those certificate policies that the signer asserts apply to the certificate, and under which the certificate should be relied upon. This value suggests a policy value to be used in the relying party's certification path validation. The definition of PolicyInformation can be found in [RFC3280].

If present, the SigningCertificateV2 attribute MUST be a signed attribute; it MUST NOT be an unsigned attribute. CMS defines SignedAttributes as a SET OF Attribute. A SignerInfo MUST NOT include multiple instances of the SigningCertificatev2 attribute. CMS defines the A.SN.1 syntax for the signed attributes to include attrValues SET OF AttributeValue. A SigningCertificateV2 attribute MUST include only a single instance of AttributeValue. There MUST NOT be zero or multiple instances of AttributeValue present in the attrValues SET OF AttributeValue.

4. Insert New Section 5.4.1.1 'Certificate Identification Version 2'

Insert the following text as a new section.

\subsubsection{Certificate Identification Version 2}

The best way to identify certificates is an often-discussed issue. The ESSCertIDv2 structure supplies two different fields that are used for this purpose.

The hash of the entire certificate allows for a verifier to check that the certificate used in the verification process was the same certificate the signer intended. Hashes are convenient in that they are frequently used by certificate stores as a method of indexing and retrieving certificates as well. The use of the hash is required by this structure since the detection of substituted certificates is based on the fact they would map to different hash values.

The issuer/serial number pair is the method of identification of certificates used in [RFC3280]. That document imposes a restriction for certificates that the issuer distinguished name must be present. The issuer/serial number pair would therefore normally be sufficient to identify the correct signing certificate. (This assumes the same issuer name is not reused from the set of trust anchors.) The issuer/serial number pair can be stored in the sid field of the SignerInfo object. However, the sid field is not covered by the signature. In the cases where the issuer/serial number pair is not used in the sid or the issuer/serial number pair needs to be signed, it SHOULD be placed in the issuerserial field of the ESSCertIDv2 structure. 


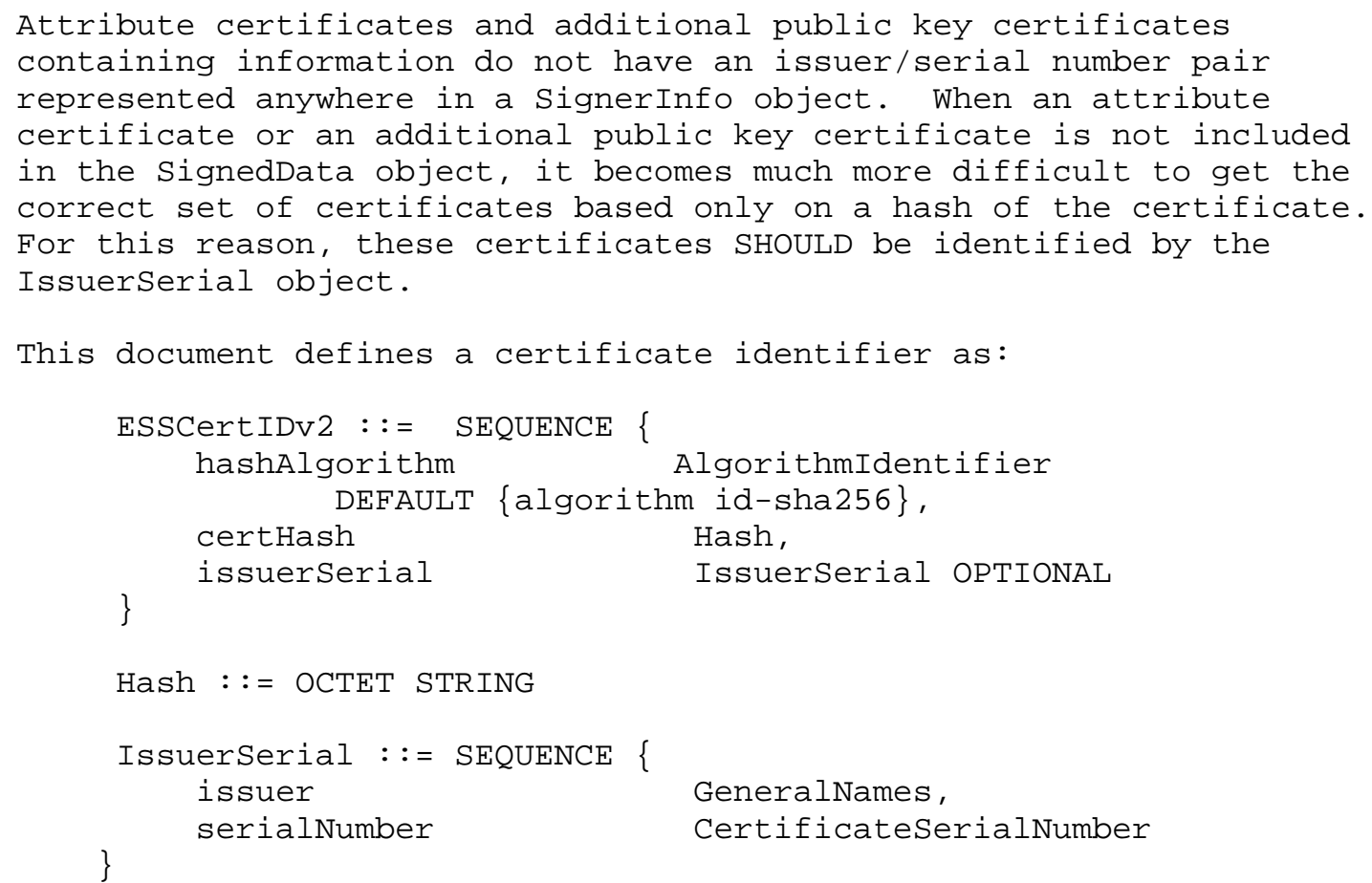

The fields of ESSCertIDv2 are defined as follows:

hashAlgorithm contains the identifier of the algorithm used in computing certHash. 
serialNumber

holds the serial number that uniquely identifies the certificate for the issuer.

5. Insert New Section 5.4.2 'Signing Certificate Attribute Definition Version $1^{\prime}$

(Note: This section does not present new material. This section contains the original contents of Section 5.4 in [ESS], which are retained with minor changes in this specification to achieve backwards compatibility.)

Insert the following text as a new section.

\subsubsection{Signing Certificate Attribute Definition Version 1}

The signing certificate attribute is designed to prevent the simple substitution and re-issue attacks, and to allow for a restricted set of certificates to be used in verifying a signature.

The definition of SigningCertificate is

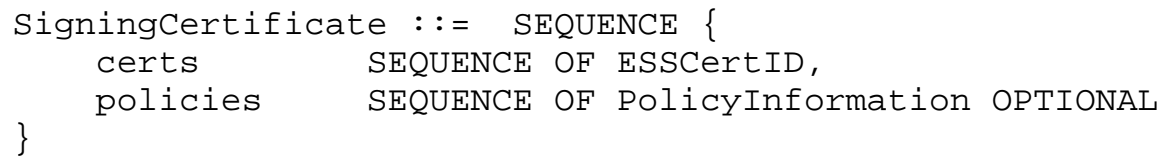

The first certificate identified in the sequence of certificate identifiers MUST be the certificate used to verify the signature. The encoding of the ESSCertID for this certificate SHOULD include the issuerserial field. If other constraints ensure that issuerAndSerialNumber will be present in the SignerInfo, the issuerSerial field MAY be omitted. The certificate identified is used during the signature verification process. If the hash of the certificate does not match the certificate used to verify the signature, the signature MUST be considered invalid.

If more than one certificate is present in the sequence of ESSCertIDs, the certificates after the first one limit the set of certificates that are used during validation. Certificates can be either attribute certificates (limiting authorizations) or public key certificates (limiting path validation). The issuerserial field (in the ESSCertID structure) SHOULD be present for these certificates, unless the client who is validating the signature is expected to have 
easy access to all the certificates required for validation. If only the signing certificate is present in the sequence, there are no restrictions on the set of certificates used in validating the signature.

The sequence of policy information terms identifies those certificate policies that the signer asserts apply to the certificate, and under which the certificate should be relied upon. This value suggests a policy value to be used in the relying party's certification path validation.

If present, the SigningCertificate attribute MUST be a signed attribute; it MUST NOT be an unsigned attribute. Cryptographic Message Syntax (CMS) defines SignedAttributes as a SET OF Attribute. A SignerInfo MUST NOT include multiple instances of the SigningCertificate attribute. CMS defines the ASN.1 syntax for the signed attributes to include attrValues SET OF AttributeValue. A SigningCertificate attribute MUST include only a single instance of AttributeValue. There MUST NOT be zero or multiple instances of AttributeValue present in the attrValues SET OF AttributeValue.

6. Insert New Section 5.4.2.1 'Certificate Identification Version 1'

(Note: This section does not present new material. This section contains the original contents of section 5.4 in [ESS], which are retained with minor changes in this specification to achieve backwards compatibility.)

Delete old Section 5.4.1

Insert the following as new text

5.4.2.1 Certificate Identification Version 1

Certificates are uniquely identified using the information in the ESSCertID structure. Discussion can be found in section 5.4.1.1.

This document defines a certificate identifier as:

$\begin{array}{cl}\text { ESSCertID }::=\text { SEQUENCE }\{ & \\ \text { certHash } & \text { Hash, } \\ \text { issuerSerial } & \text { Issuerserial OPTIONAL }\end{array}$


The fields of ESSCertID are defined as follows:

certHash

is computed over the entire DER-encoded certificate (including the signature).

issuerSerial

holds the identification of the certificate. This field would normally be present unless the value can be inferred from other information (e.g., the sid field of the SignerInfo object).

The fields of IssuerSerial are discussed in Section 5.4.1.1

7. Security Considerations

This document is designed to address the security issue of a substituted certificate used by the validator. If a different certificate is used by the validator than the signer, the validator may not get the correct result. An example of this would be that the original certificate was revoked and a new certificate with the same public key was issued for a different individual. Since the issuer/ serial number field is not protected, the attacker could replace this and point to the new certificate and validation would be successful.

The attributes defined in this document are to be placed in locations that are protected by the signature. This attribute does not provide any additional security if placed in an unsigned or un-authenticated location.

The attributes defined in this document permit a signer to select a hash algorithm to identify a certificate. A poorly selected hash algorithm may provide inadequate protection against certificate substitution or result in denial of service for this protection. By employing the attributes defined in this specification with the same hash algorithm used for message signing, the sender can ensure that these attributes provide commensurate security.

Since recipients must support the hash algorithm to verify the signature, selecting the same hash algorithm also increases the likelihood that the hash algorithm is supported in the context of certificate identification. Note that an unsupported hash algorithm for certificate identification does not preclude validating the message but does deny the message recipient protection against certificate substitution.

To ensure that legacy implementations are provided protection against certificate substitution, clients are permitted to include both ESScertID and ESScertIDv2 in the same message. Since these 
attributes are generated and evaluated independently, the contents could conceivably be in conflict. Specifically, where a signer has multiple certificates containing the same public key, the two attributes could specify different signing certificates. The result of signature processing may vary depending on which certificate is used to validate the signature.

Recipients that attempt to evaluate both attributes may choose to reject such a message.

8. Normative References

[ESS] Hoffman, P., "Enhanced Security Services for S/MIME", RFC 2634, June 1999.

[RFC2119] Bradner, S., "Key words for use in RFCs to Indicate Requirement Levels", RFC 2119, BCP 14, March 1997.

[RFC3280] Housley, R., Ford, W., Polk, W., and D. Solo, "Internet X.509 Public Key Infrastructure Certificate and Certificate Revocation List (CRL) Profile", RFC 3280, April 2002 .

[RFC3852] Housley, R., "Cryptographic Message Syntax (CMS) ", REC 3852, July 2004 .

[UTF8] Yergeau, F., "UTF-8, a transformation format of ISO 10646", STD 63, RFC 3629, November 2003. 
Appendix A. ASN.1 Module

Replace the ASN.1 module in RFC 2634 with this one.

ExtendedSecurityServices-2006

\{ iso(1) member-body(2) us(840) rsadsi(113549)

pkcs (1) pkcs-9(9) smime(16) modules(0) id-mod-ess-2006(30) \}

DEFINITIONS IMPLICIT TAGS : :=

BEGIN

IMPORTS

-- Cryptographic Message Syntax (CMS) [RFC3852]

ContentType, IssuerAndSerialNumber, SubjectKeyIdentifier

FROM CryptographicMessageSyntax2004 \{ iso(1) member-body (2)

us (840) rsadsi(113549) pkcs(1) pkcs-9(9) smime(16) modules (0) cms-2004(24)\}

-- PKIX Certificate and CRL Profile, Section A.1 Explicity Tagged Module

-- 1988 Syntax [RFC3280]

AlgorithmIdentifier, CertificateSerialNumber

FROM PKIX1Explicit88 \{ iso(1) identified-organization(3) dod(6) internet(1) security (5) mechanisms (5) pkix(7) id-mod(0)

id-pkixl-explicit(18) \}

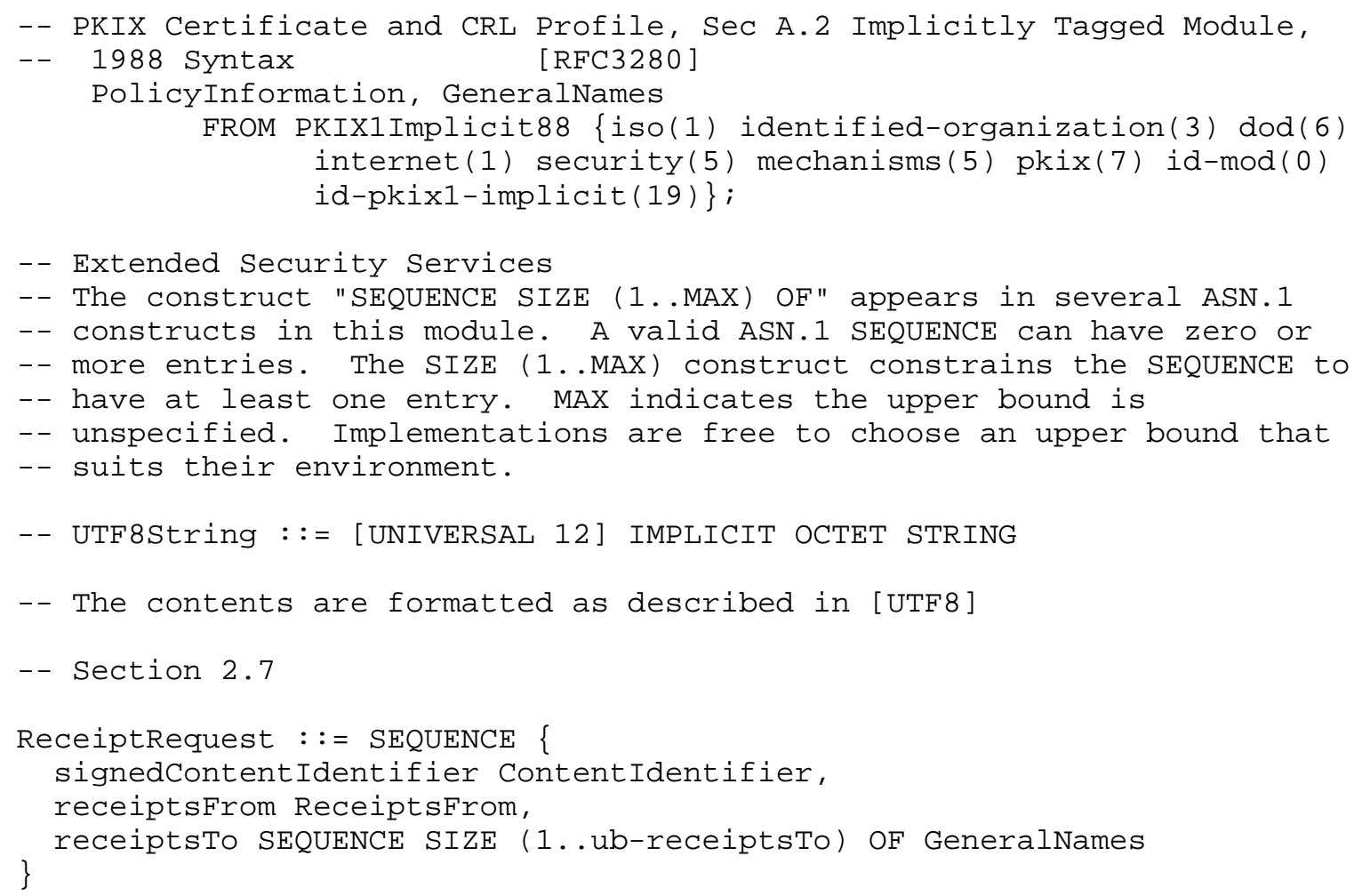




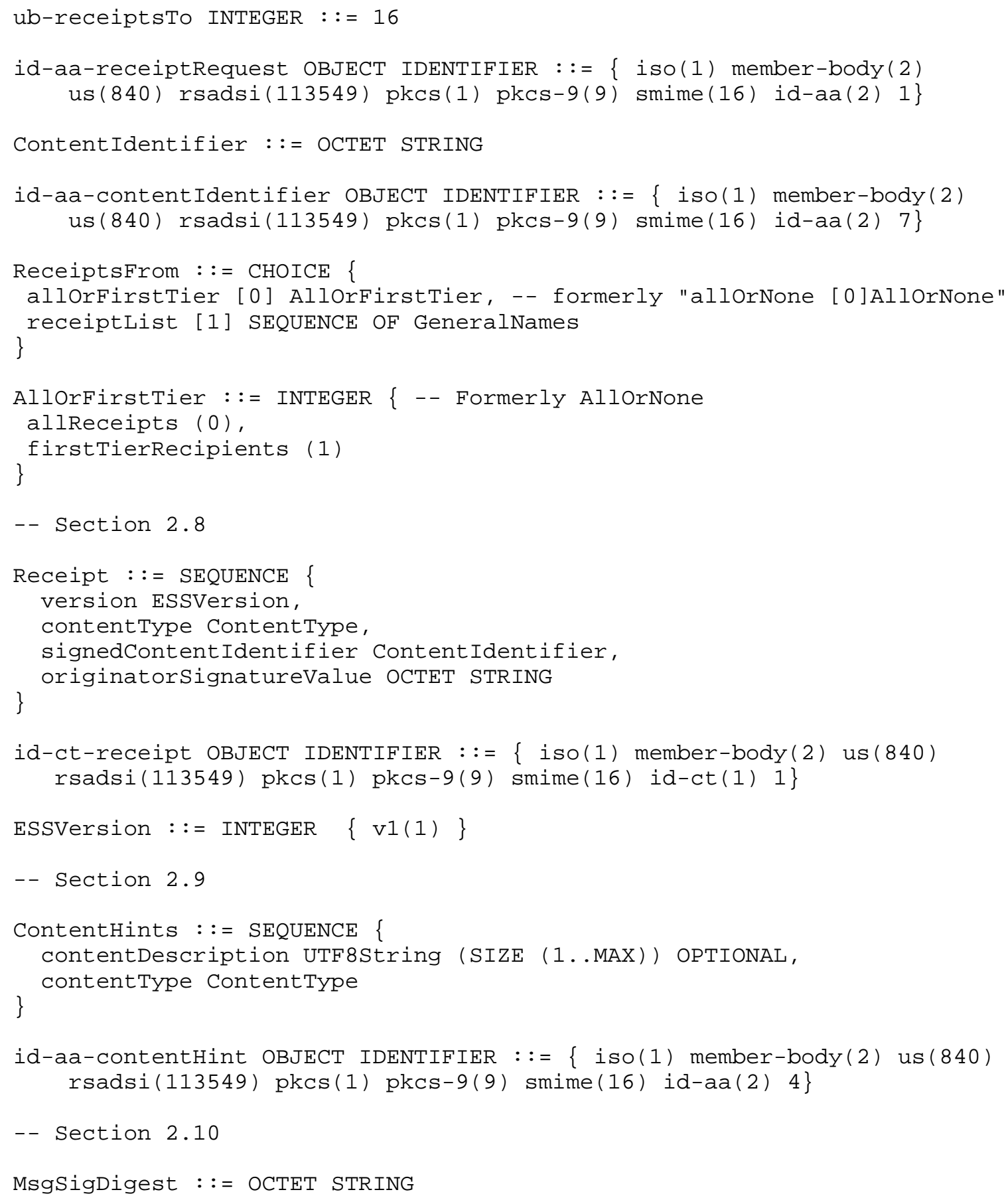




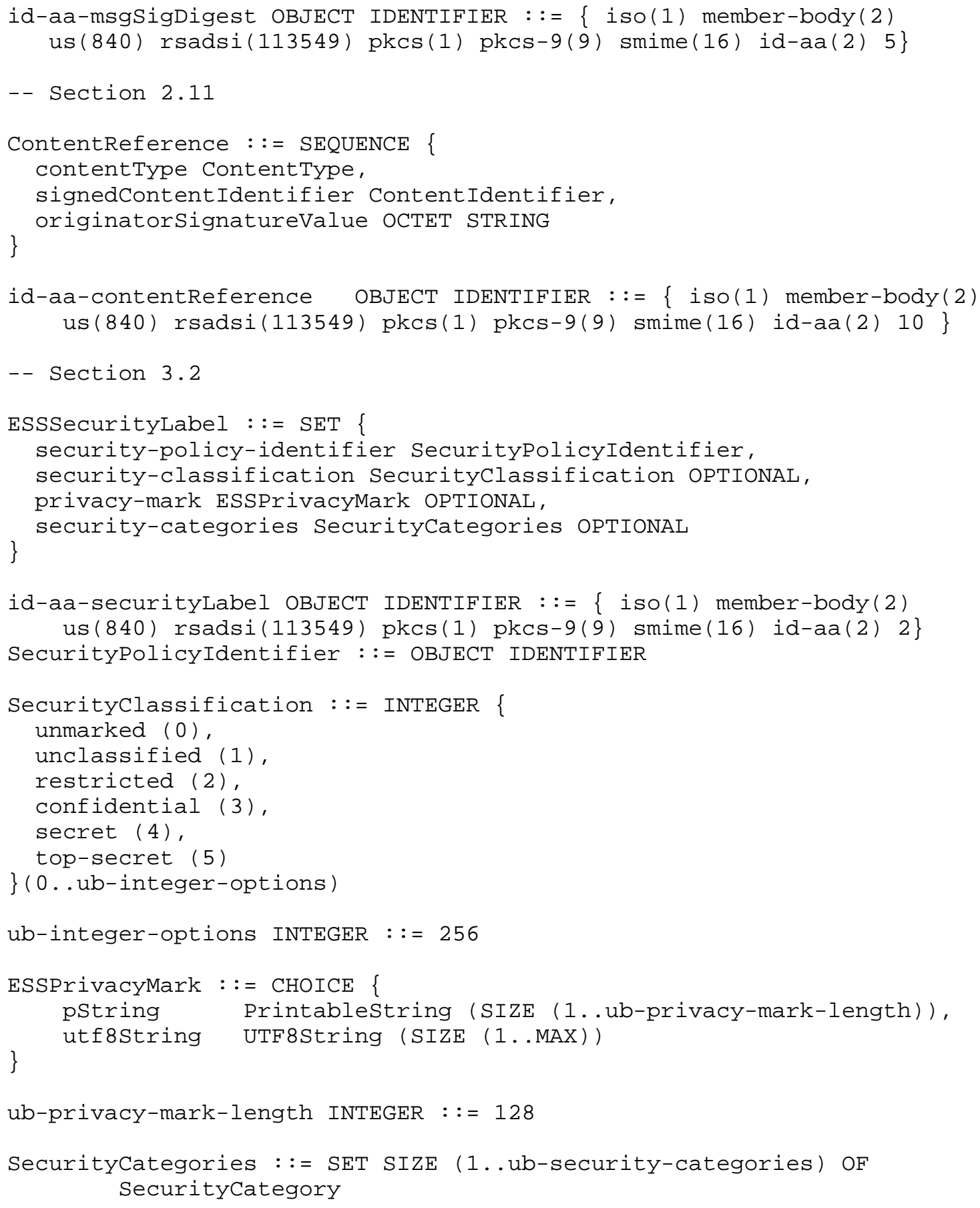




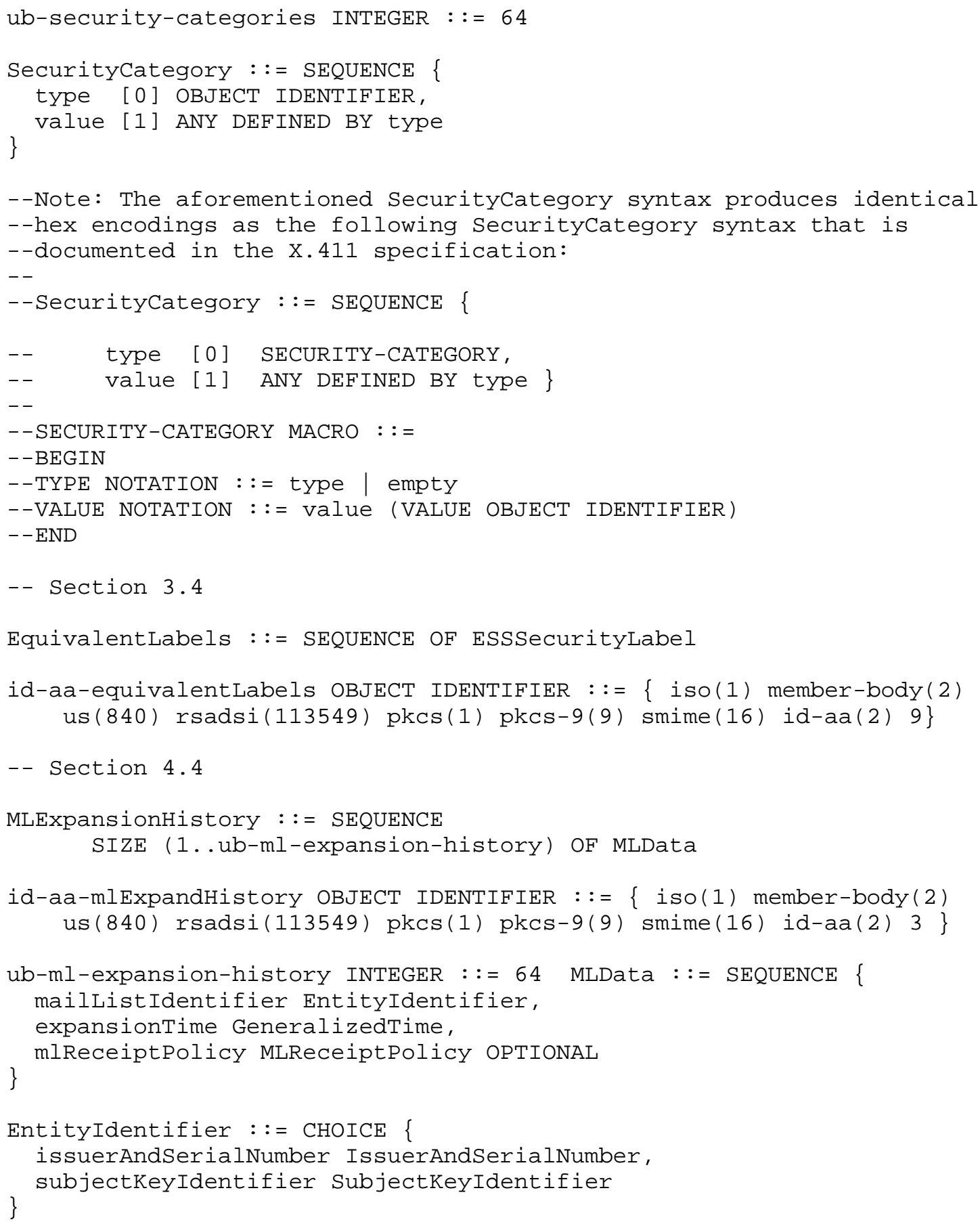




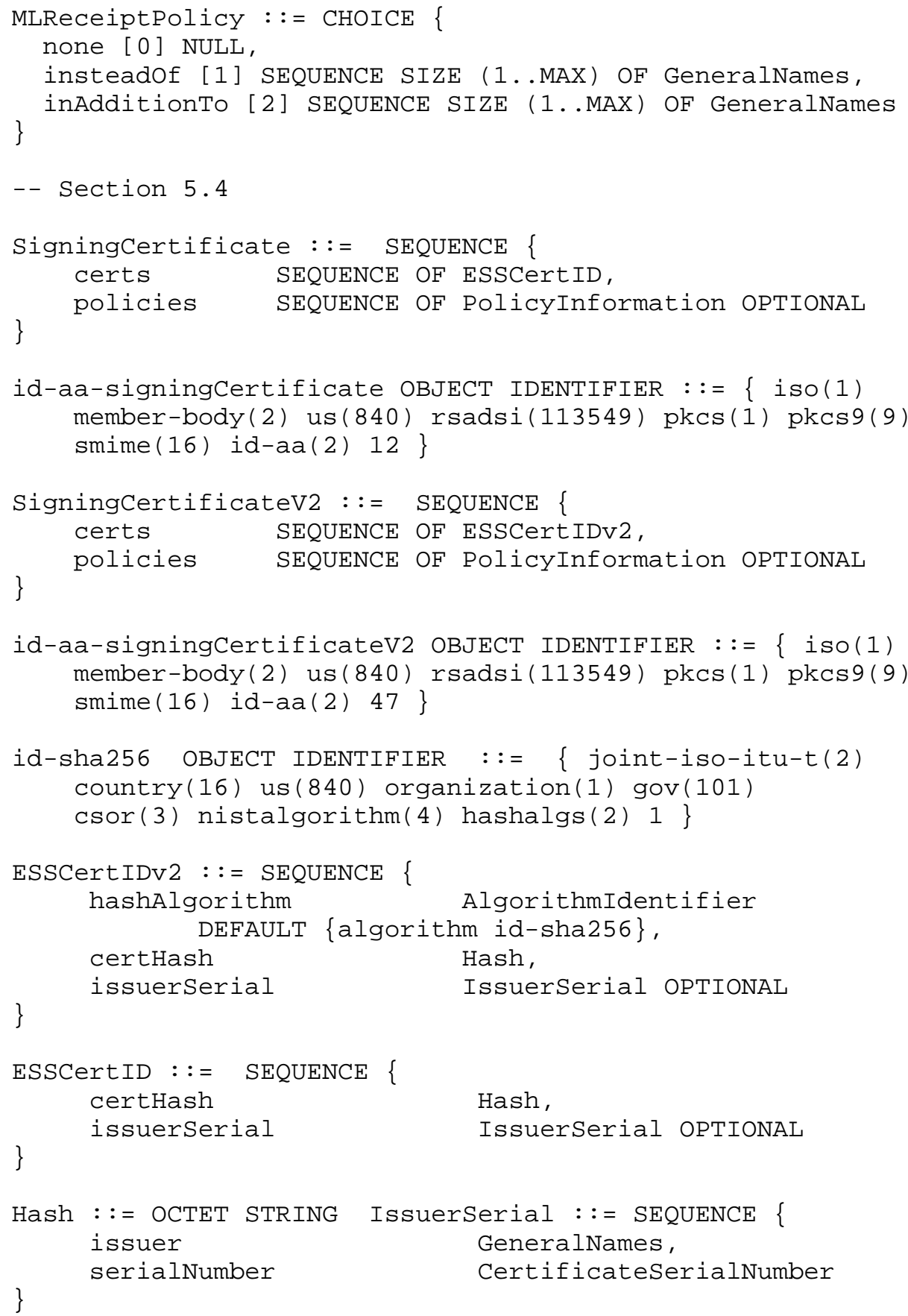


-- of ExtendedSecurityServices-2006

Author's Address

Jim Schaad

Soaring Hawk Consulting

PO Box 675

Gold Bar, WA 98251

EMail: jimsch@exmsft.com 
Full Copyright statement

Copyright (C) The IETF Trust (2007).

This document is subject to the rights, licenses and restrictions contained in BCP 78, and except as set forth therein, the authors retain all their rights.

This document and the information contained herein are provided on an "AS IS" basis and THE CONTRIBUTOR, THE ORGANIZATION HE/SHE REPRESENTS OR IS SPONSORED BY (IF ANY), THE INTERNET SOCIETY, THE IETF TRUST AND THE INTERNET ENGINEERING TASK FORCE DISCLAIM ALL WARRANTIES, EXPRESS OR IMPLIED, INCLUDING BUT NOT LIMITED TO ANY WARRANTY THAT THE USE OF THE INFORMATION HEREIN WILL NOT INFRINGE ANY RIGHTS OR ANY IMPLIED WARRANTIES OF MERCHANTABILITY OR FITNESS FOR A PARTICULAR PURPOSE.

Intellectual Property

The IETF takes no position regarding the validity or scope of any Intellectual Property Rights or other rights that might be claimed to pertain to the implementation or use of the technology described in this document or the extent to which any license under such rights might or might not be available; nor does it represent that it has made any independent effort to identify any such rights. Information on the procedures with respect to rights in RFC documents can be found in BCP 78 and BCP 79 .

Copies of IPR disclosures made to the IETF Secretariat and any assurances of licenses to be made available, or the result of an attempt made to obtain a general license or permission for the use of such proprietary rights by implementers or users of this specification can be obtained from the IETF on-line IPR repository at http://www.ietf.org/ipr.

The IETF invites any interested party to bring to its attention any copyrights, patents or patent applications, or other proprietary rights that may cover technology that may be required to implement this standard. Please address the information to the IETF at ietf-ipreietf.org. 